\title{
Computational study of metallic dopant segregation and embrittlement at Molybdenum grain boundaries
}

\author{
Richard Tran, Zihan Xu, Naixie Zhou, Balachandran Radhakrishnan, Jian \\ Luo, Shyue Ping Ong* \\ Department of NanoEngineering, University of California San Diego, La Jolla, CA 92093, \\ USA
}

\begin{abstract}
Mo and its alloys have been widely used as refractory materials owing to their excellent high temperature properties, but a critical limitation is their low ductility. Doping the grain boundaries (GBs) of Mo with metals such as $\mathrm{Zr}$ or $\mathrm{Al}$ have previously been demonstrated as a promising approach to address this shortcoming, whereas other alloy elements are known to embrittle the GBs. In this work, we investigated the segregation and strengthening/embrittling effects of 29 metallic dopants at the $\Sigma 5$ (310) tilt and $\Sigma 5$ (100) twist Mo GBs using density functional theory (DFT) calculations and empirical continuum models. In agreement with previous works for other metals, we find that the strain, as measured by the relative metallic radius versus Mo, is a good predictor of the segregation tendency, while the difference in cohesive energies between the dopant and Mo is a good predictor of the strengthening/embrittling effect. However, we find that dopant chemistry also plays a significant role in affecting segregation behavior at GBs, particularly in driving the formation of intermetallic precipitates or 2-D interfacial phases (complexions). We also show that the site preference of a dopant in the GB can lead to strengthening effects that deviate from those predicted using simple bond-breaking arguments. Assuming a fast cleavage model of fracture, Ta, Re, Os and $\mathrm{W}$ are predicted to have a weak strengthening effect on Mo for the $\Sigma 5(310)$ tilt GB, and Mn, Fe, Co and $\mathrm{Nb}$ are predicted to have reasonable strengthening effects for the $\Sigma 5(100)$ twist GB.
\end{abstract}

Keywords: DFT, Embrittlement, Strengthening, Grain boundary segregation, Molybdenum

\footnotetext{
* Corresponding author

Email address: ongsp@ucsd.edu (Shyue Ping Ong)
}

Preprint submitted to Acta Materialia

June 13, 2016

(C) 2016. This manuscript version is made available under the Elsevier user license http://www.elsevier.com/open-access/userlicense/1.0/ 


\section{Introduction}

Mo [1] and its alloys [2] have been widely used in high temperature applications [3] owing to their excellent stability, strength, high thermal and electrical conductivity [4], and a low coefficient of thermal expansion [5]. In addition, its

5 high creep, corrosion, and sputtering resistance opens up applications in medical devices [6] and coatings for aerospace and defense components [7]. However, Mo still suffers from low ductility at near room temperatures $[8,9]$.

It is well known that segregation of alloying elements or impurities at grain boundaries (GBs) leads to embrittling effects in Mo as well as many other al-

10 loys $[10,11,12,13,14]$. Nonetheless, doping with certain other elements can also be a promising approach to mitigate the low ductility of Mo, as demonstrated by Miller et al. [15] for $\mathrm{Zr}, \mathrm{Al}, \mathrm{C}$ and B dopants in 2002 Charai et al. [16]. also investigated liquid metal wetting embrittlement (LME) of $\mathrm{Pb}$, $\mathrm{Sn}$ and $\mathrm{Ni}$ on $\mathrm{Mo}$ GBs and showed that the wettability by $\mathrm{Pb}$ and $\mathrm{Ni}$ is dependent on crystal-

15 lography or GB types. More recently Liu et al. [8], reported a nanostructuring technique that involves a molecular-level liquid-liquid mixing/doping to achieve yield strengths over $800 \mathrm{MPa}$ and tensile elongation as large as $40 \%$ at room temperature in Mo alloys. On the computational front, first principles investigations of dopant segregation and diffusion in Mo GBs have been carried out with

20 light elemental dopants such as B, C, N, O and H [17, 18]. In particular Janisch and Elsässer [17], used local-density functional theory (LDFT) to confirm one of the predictions made by the empirical Cotrell model [19] by showing that the bonding strength between Mo GBs and its interstitial dopants depends on the strength of hybridization due to the relative position of electronic bands and 25 thus on the valence configuration of the elements.

GBs in metals critically affect their mechanical properties by acting as centers of dopant segregation [20,21] and system stabilization [22]. Although GB segregation in other metals, such as Fe [23], W [24], and Ni [25, 26], have been extensively studied, a systematic first principles investigation of metallic dopant segregation in Mo GBs is yet to be carried out.

In this work, we performed density functional theory (DFT) calculations to investigate segregation and strengthening phenomena in the $\Sigma 5(310)$ symmetric tilt and $\Sigma 5(100)$ twist GBs of Mo for 29 metallic dopants. We compared the results of our investigation with those computed from the empirical Miedema [27]

35 and McLean [28] models. We will discuss whether segregation and strengthening/embrittling effects can be explained using simple models based on strain and cohesive energy, and the reasons for observed deviations from these models.

\section{Methods}

For brevity, the terms "twist GB" and "tilt GB" will henceforth be used

40 to refer to the $\Sigma 5(100)$ twist and $\Sigma 5(310)$ tilt GBs investigated in this work. All GB model construction, input file generation and analyses were performed using the Python Materials Genomics (pymatgen) library [29]. The following subsections outline the various structural models and calculation parameters 
used. Comprehensive convergence tests were carried out (see Figure S1 in Supplementary Information) to ensure that the relevant properties of interest (e.g., segregation energy) were converged to within $0.02 \mathrm{eV}$ using these models and parameters.

\subsection{GB structural models}

GB structures were generated from the fully relaxed body-centered cubic (bcc) Mo unit cell with cubic lattice parameter $a=3.167 \AA$. The tilt GB model (Figure 1(a)) had dimensions $9.443 \AA \times 9.956 \AA \times 24.760 \AA$ with 144 atoms, and the twist GB model (Figure 2 ) had dimensions $7.083 \AA \times 7.083 \AA \times 25.343 \AA$ with 80 atoms. The twist GB model was generated in accordance to coincidental site lattice (CSL) theory [30]. Free surface calculations were performed using cells of 55 the same cell sizes as the GB models, but with approximately half the numbers of atoms.

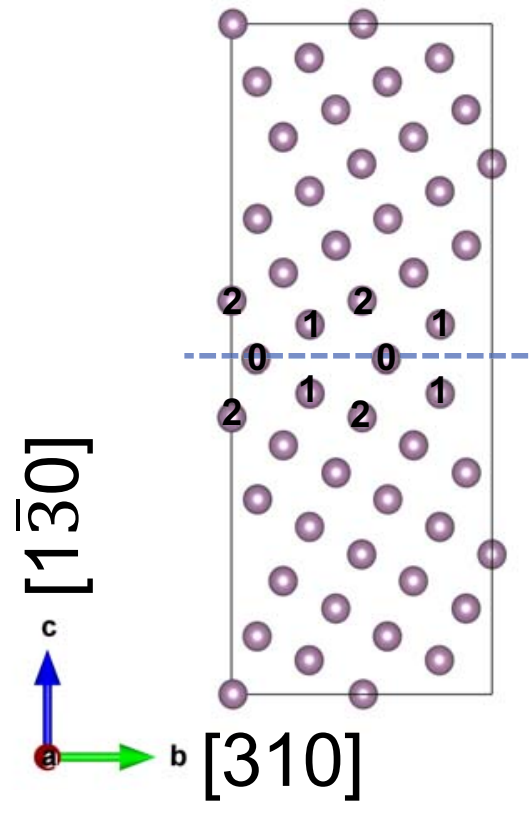

(a) View along $a$ axis.

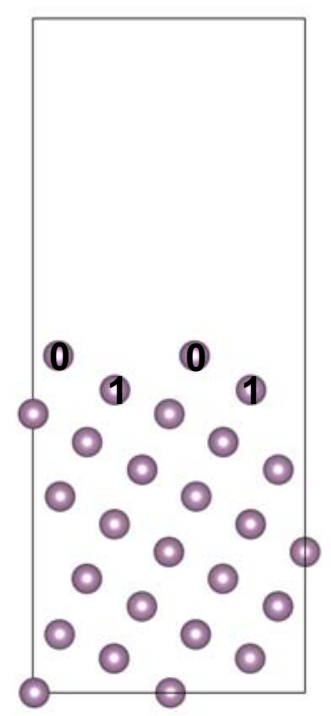

(b) (310) surface slab.

Figure 1: Structure model for (a) the Mo $\Sigma 5(310)$ tilt GB and (b) the Mo (310) surface slab. Symmetrically distinct sites in the tilt GB are numbered with increasing integers representing increasing distance from the mirror plane. 


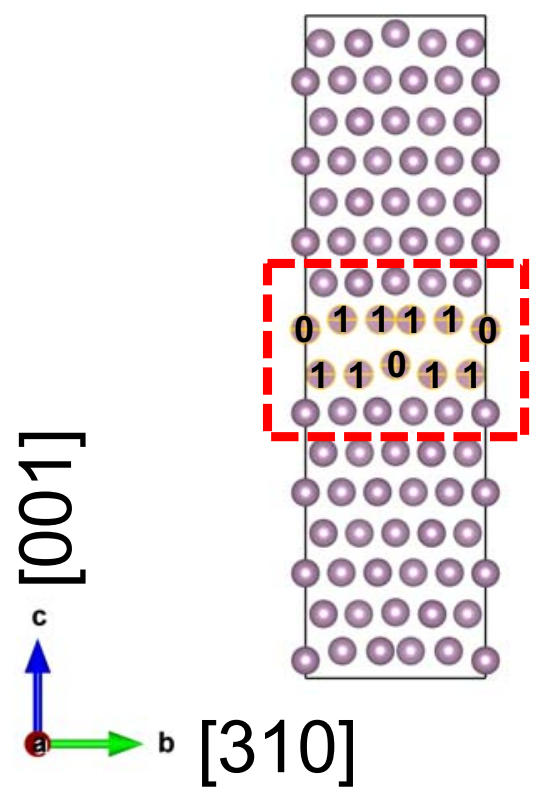

(a) View along $a$ axis.

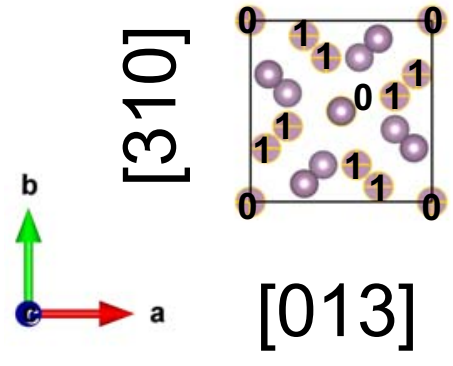

(b) View of red rectangular region along $c$ axis.

Figure 2: Structure model for Mo $\Sigma 5(100)$ twist GB. Coincident sites are labeled with the number 0 , while non-coincident sites are labeled as 1 . 


\subsection{DFT calculations}

All DFT calculations [31, 32] were performed using the Vienna Ab initio Simulation Package (VASP) [33] within the projector augmented wave (PAW) [34]

60 approach. The exchange-correlation effects were modeled using the PerdewBerke-Ernzerhof (PBE) generalized gradient approximation (GGA) [35] functional, and all calculations were spin-polarized. A plane wave cutoff energy of $400 \mathrm{eV}$ and a Gamma-centered $k$-point mesh were used for all calculations. The energies and atomic forces of all calculations were converged to within $5 \times 10^{-4}$

$65 \mathrm{eV}$ and $0.02 \mathrm{eV}^{-1}$ respectively. The Methfessel-Paxton method [36] was chosen as the smearing algorithm, the blocked Davidson iteration scheme [37] was chosen as the electron minimization algorithm, and ions were updated with the conjugated gradient algorithm. $\Gamma$-centered $k$-point meshes of $6 \times 6 \times 1$ and $7 \times 7 \times 1$ were chosen for the tilt and twist boundary structures, respectively.

\subsection{Key parameters for segregation studies}

From DFT calculations, the grain boundary (GB) and free surface (FS) energies are given by the following equation:

$$
\gamma_{G B / F S}=\frac{E_{G B / F S}-E_{\text {bulk/atom }} \times n_{G B / F S}}{2 \times A_{G B / F S}}
$$

where $\gamma_{G B / F S}$ is the GB/FS energy, $E_{G B / F S}$ is the energy of the supercell containing the GB or FS, and $E_{b u l k / \text { atom }}$ is bulk energy per atom in the Mo bcc structure, $A_{G B / F S}$, is the cross-section area and $n_{G B / F S}$ is the number of atoms in the GB/FS supercell.

The propensity of the dopant to segregate into the GB/FS is characterized by its segregation energy $E_{\text {seg }}^{G B / F S}$, which is given by the relative difference in energy between the dopant in the GB/FS and in the bulk. The segregation energy of a dopant $X$ can be computed using the following equation:

$$
E_{\text {seg }}^{G B / F S}=\left(E_{G B / F S+X}-E_{G B / F S}\right)-\left(E_{b u l k+X}-E_{b u l k}\right)
$$

75 where $E_{G B / F S / b u l k+X}$ is the energy for the doped GB/FS/bulk structure and $E_{G B / F S / b u l k}$ is the energy of the undoped GB/FS/bulk structure. In this work, all doped structures were obtained by substituting a single Mo atom with a dopant atom in one of the equivalent sites giving a dopant area density $\left(\Gamma_{G B}\right)$ of $0.020 \AA^{-2}$ and $0.012 \AA^{-2}$ for the twist and tilt GBs respectively. The atomic positions were then relaxed with the lattice parameters fixed at those of the fully-relaxed undoped structures. Interstitial doping was not investigated. A negative $E_{\text {seg }}^{G B / F S}$ indicates dopant segregation into the GB/FS is energetically favorable, in line with the convention used in literature [24, 25, 26, 23].

From $E_{\text {seg }}^{G B}$ and $E_{\text {seg }}^{F S}$, the strengthening energy, $E_{S E}$ can be obtained from the following equation

$$
E_{S E}=E_{s e g}^{G B}-E_{s e g}^{F S}=\left(E_{G B+X}-E_{G B}\right)-\left(E_{F S+X}-E_{F S}\right)
$$


A positive/negative $E_{S E}$ indicates that the grain boundary is weakened/strengthened by the dopant. It should be noted that $E_{S E}$ is equivalent to the difference in work of fracture $\left[w_{\text {sep }}=\gamma_{G B}-2 \gamma_{F S}\right]$ [38] between the doped and undoped grain boundaries. In this work, only one dopant area density was investigated for the twist and tilt GBs, though the coverage of the dopant on the GB/FS would no doubt have an effect on the work of separation. [38] A detailed discussion of 90 these measures can be found in Razumovskiy et al. [25].

\subsection{Empirical continuum models}

In this work, we compared the DFT predictions with two empirical GB thermodynamic models: the McLean model [28] and the Miedema model [27]. The McLean model only considers strain energy of the solute, which in this case is derived from the Friedel model [39], as the major factor in GB segregation, and the segregation enthalpy $\left(\Delta \mathrm{H}^{\text {seg }}\right)$ is given by the following equation:

$$
\Delta H_{e l}^{s e g}=\frac{24 \pi K_{\mathrm{Mo}} G_{\mathrm{X}} r_{\mathrm{Mo}} r_{\mathrm{X}}\left(r_{\mathrm{Mo}}-r_{\mathrm{X}}\right)^{2}}{3 K_{\mathrm{Mo}} r_{\mathrm{Mo}}+4 G_{\mathrm{X}} r_{\mathrm{X}}}
$$

where $K$ and $G$ are the bulk modulus and shear modulus, respectively, the subscript $\mathrm{X}$ denoting the dopant, and $r$ is the atomic radius.

The Miedema model posits that the bonding energy differences between dopant and bulk atoms contribute to GB segregation enthalpy, with $\Delta \mathrm{H}^{s e g}$ given by the following equation:

$$
\Delta H^{\text {seg }}=-0.71 \times \frac{1}{3} \times \nu \times\left(-\Delta H_{\mathrm{sol}}^{\mathrm{Mo}} c_{0} \gamma_{\mathrm{Mo}}^{S} V_{\mathrm{Mo}}^{2 / 3}+c_{0} \gamma_{\mathrm{X}}^{S} V_{\mathrm{X}}^{2 / 3}\right)+\Delta E_{e l}
$$

where $\nu$ is the ratio of lost bonds at GB core, $c_{0} \gamma^{S} V^{2 / 3}$ represents the molar 95 surface enthalpy of pure metal (Mo or X) by the definition of Miedema, $\Delta H_{\mathrm{sol}}^{\mathrm{Mo}}$ is the enthalpy of solution of $\mathrm{X}$ in Mo and $\Delta E_{e l}$ is the solute strain energy.

Because the empirical continuum models assume that changes in volume and entropy due to segregation are negligible, segregation energy $E_{\text {seg }}^{G B}$ is approximately equal to segregation enthalpy, we will use the former term in the re-

100 mainder of this article for consistency. Also, $E_{\text {seg }}^{G B}$ calculated using the empirical models assume the reverse convention whereby a positive value indicates segregation preference towards the GB while a negative value indicates the dopant tends to stay in the bulk. To maintain consistency when comparing to the DFT values, $E_{\text {seg }}^{G B}$ derived from these models will be presented with the reverse sign.

\section{Results}

Given the relatively large number of dopants explored in this work, we have adopted a consistent marker shape and color scheme for all figures for ease of reference. The marker type indicates the type of dopant (circle: $3 d$ transition metal; square: $4 d$ transition metal; triangle: $5 d$ transition metal; pentagon:

110 other $s / p$ metals), and the marker colors are assigned in order of increasing atomic number within each group of dopants, i.e., for the transition metals, dopants in the same periodic group share the same color. 


\subsection{Site preference for dopants in Mo twist and tilt GBs}

For both the tilt and twist GBs, there are multiple distinct sites close to the GBs that may be potentially occupied by the dopant:

1. In the tilt GB, we considered three distinct sites: the site lying on the mirror plane (Site 0) and the two sites that are nearest to the mirror plane (Sites 1 and 2), as shown in Figure 1(a). As seen in Table 1, Sites 0 and 2 have larger average nearest neighbor distances and coordination numbers than Site 1. The polyhedron volume of Site 1 is the smallest among the three sites.

2. In the twist GB, we investigated two distinct sites: (i) the coincidental site (Site 0) and (ii) the non-coincidental site (Site 1), as shown in Figure 2. As seen in Table 1 , Site 0 has a larger coordination number, average nearest neighbor distance, and polyhedron volume than Site 1.

Figure 3 plots the energy differences between GBs doped at different sites against the relative metallic radius $R_{X} / R_{M o}$ of the dopant. We find that larger dopants generally prefer larger sites (Site 0 in the twist GB and Sites 0 and 2 in the tilt $\mathrm{GB}$ ), and vice versa. In the tilt GB, dopants with radii smaller than Mo tend to favor the site just above the mirror plane (Site 1), while dopants with radii larger than Mo show a clear preference for the mirror plane site (Site 0), as shown in Figures 3(a). An outlier is Pt, which shows a strong preference for the larger Sites 0 and 2 relative to the smaller Site 1 despite having a metallic radius slightly smaller than Mo. None of the dopants prefer Site 2 over Site 135 0. For the twist GB (Figure 3(c)), dopants with radii smaller than Mo clearly prefer the non-coincidental site (Site 1) while dopants with radii larger than Mo present little to no site preference.

\begin{tabular}{lccccc}
\hline & $\Sigma 5(100)$ & Twist & \multicolumn{3}{c}{$\Sigma 5(310)$ Tilt } \\
Site & 0 & 1 & 0 & 1 & 2 \\
\hline Coordination number & 8 & 6 & 7 & 8 & 8 \\
Average bond length $(\AA)$ & 2.835 & 2.667 & 2.840 & 2.717 & 2.747 \\
Polyhedron volume $\left(\AA^{3}\right)$ & 39.041 & 20.116 & 31.976 & 26.726 & 31.869 \\
\hline
\end{tabular}

Table 1: The coordination number, average bond length and polyhedron volume for each site before dopant insertion.

\subsection{Dopant segregation energy}

Figure 4 plots the segregation energy for the lowest energy sites of the twist GB $E_{\text {seg }}^{\mathrm{X} \text {, twist }}$ against that for the tilt GB $E_{\text {seg }}^{\mathrm{X} \text {, tilt }}$. In general, we find that the segregation energies of most dopants to either type of GB are within $\pm 0.25 \mathrm{eV}$ of each other, with the exception of the magnetic $3 d$ transition metals, $\mathrm{Pd}$ and Pt. Of the 29 dopants investigated, only W, Ti and V are predicted to have a small positive $E_{\text {seg }}^{\mathrm{X} \text {, twist }}$, and only $\mathrm{Ti}$ is predicted to have a small positive $E_{\text {seg }}^{\mathrm{X} \text {, tilt }}$. 145 All other dopants are predicted to have negative segregation energy for both 


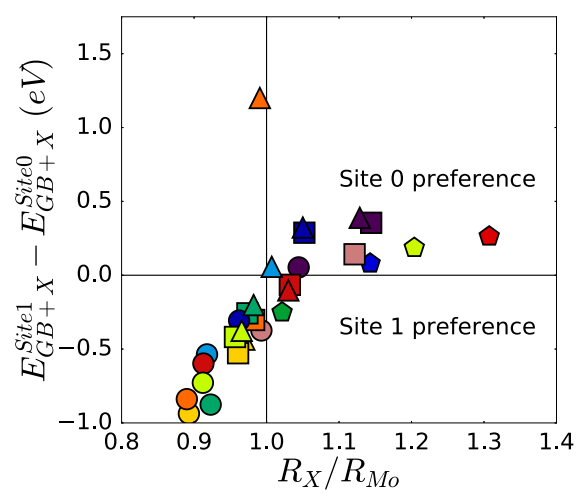

(a) $\Sigma 5$ (310) Tilt: Site 1 vs Site 0

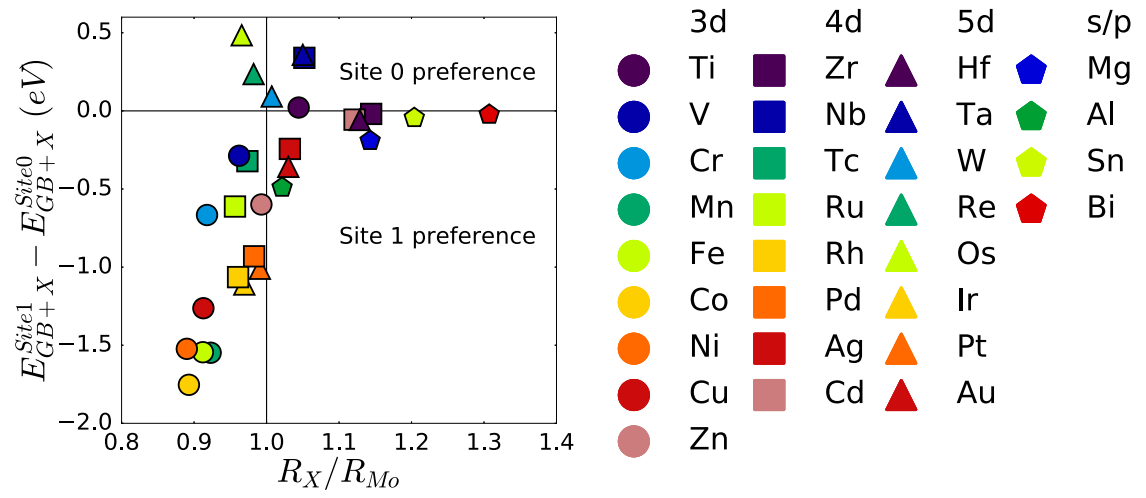

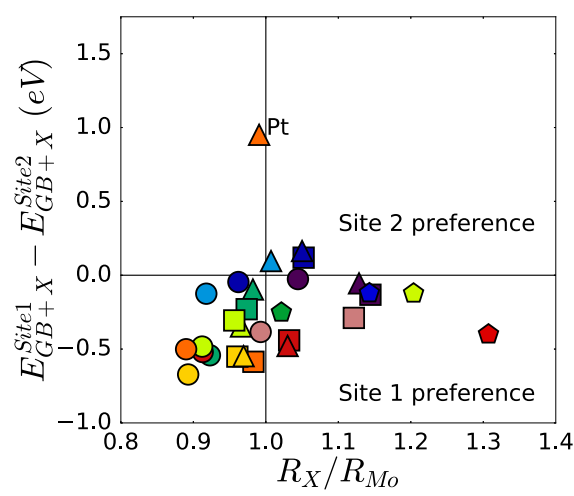

(b) $\Sigma 5$ (310) Tilt: Site 1 vs Site 2

(c) $\Sigma 5$ (100) Twist: Site 1 vs Site 0 
types of GBs, i.e., there is a thermodynamic driving force towards segregation. Given that $E_{\text {seg }}^{\mathrm{X} \text {, twist }}$ and $E_{\text {seg }}^{\mathrm{X} \text {, tilt }}$ are relatively similar, and the tilt GB has a lower calculated energy $\left(\gamma_{\text {tilt }}=1.83 \mathrm{Jm}^{-2}\right)$ than the twist $\mathrm{GB}\left(\gamma_{\text {twist }}=2.43\right.$ $\mathrm{Jm}^{-2}$ ), we will henceforth present mainly the results for the $\Sigma 5(310)$ tilt GB and use $E_{\text {seg }}^{\mathrm{X}}$ to refer to $E_{\text {seg }}^{\mathrm{X} \text {, tilt }}$ for brevity. Interested readers are referred to the Supplementary Information for the corresponding plots and results for the $\Sigma 5(100)$ twist GB.

Figure 5 compares the DFT segregation energy for all dopants in the $\Sigma 5(310)$ tilt GB with those predicted from the empirical McLean and Miedema models.

155 Again, we note here that we have inverted the sign of the $E_{\text {seg }}^{\mathrm{X}}$ from the empirical models such that a negative $E_{\text {seg }}^{\mathrm{X}}$ always implies a driving force to segregate. We observe that both empirical models tend to predict $E_{\text {seg }}$ that are less negative compared to DFT. In particular, the $E_{\text {seg }}^{\mathrm{X}}$ of the noble metals (Rh, Ir, Pt, Ru, $\mathrm{Pd}, \mathrm{Ag}, \mathrm{Au}$ ), $3 d$ transition metals (Co, Ni, Fe), Re, Os and Tc predicted by both empirical models are much less negative than that predicted in DFT. On the other hand, $\mathrm{Mg}$ and $\mathrm{Ti}$ are both predicted by the empirical models to have more negative $E_{\text {seg }}^{\mathrm{X}}$ compared to the DFT predictions.

Figure $6(\mathrm{a})$ plots $E_{\text {seg }}^{\mathrm{X}}$ against normalized difference in metallic radii $\frac{R_{X}-R_{M o}}{R_{M o}}$ for all dopants. We may observe that there is a somewhat parabolic-like relationship between $E_{\text {seg }}^{\text {tilt }}$ and relative metallic radii, which indicates that the strain energy is likely a significant contributing factor to segregation. However, the noble metals and $3 d$ transition metals are again outliers, predicted by DFT to have relatively large $E_{\text {seg }}^{\mathrm{X}}$, despite having relatively small metallic radii mismatch with Mo.

${ }_{170} \quad$ Figure $6(\mathrm{~b})$ plots $E_{\text {seg }}^{\mathrm{X}}$ against the square of the Pauling electronegativity difference $\left(\chi_{\mathrm{X}}-\chi_{\mathrm{Mo}}\right)^{2}$ between each dopant and Mo, which is proportional to the difference in the dissociation energy of the dopant $\mathrm{X}$ and the average dissociation energy of $\mathrm{X}$ and Mo [40]. We find that there is a reasonably strong monotonic relationship between $E_{\text {seg }}^{\mathrm{X}}$ and $\left(\chi_{\mathrm{X}}-\chi_{\mathrm{Mo}}\right)^{2}$, with increasing electronegativity difference generally leading to less negative $E_{\text {seg }}^{\mathrm{X}}$.

\subsection{Strengthening energy}

In Figure 7, the strengthening energy $E_{S E}^{\mathrm{X}}$ is plotted against the segregation energy $E_{\text {seg }}^{\mathrm{X}}$ for the 29 dopants in the $\Sigma 5$ (310) tilt GB. The dopants can be classified into three zones of interest: dopants that have positive $E_{\text {seg }}^{\mathrm{X}}$ (white 180 region) would prefer to stay in the bulk, and hence would have no effect on GB strength. For dopants that segregate, those with negative $E_{S E}^{\mathrm{X}}$ (blue region) tend to strengthen the GB while those with positive $E_{S E}^{\mathrm{X}}$ tend to embrittle it.

For Figure $7(\mathrm{a}), E_{S E}^{\mathrm{X}}$ and $E_{\text {seg }}^{\mathrm{X}}$ were determined based on the lowest energy dopant site in the GB and FS (henceforth, referred to as the "l-to-l" approach), while for Figure 7(b), they were determined based on Site 0 in both the GB and FS, i.e., the mirror plane site in the GB and surface site in the FS (henceforth, referred to as the "m-to-s" approach). We find that the site preference has a significant influence on the DFT predictions. Using the l-to-l approach, the DFT calculations predict that Pd, Cu, Ta, Cr, Tc, W, Fe, Ni, Ru, Rh, Co, Mn, 


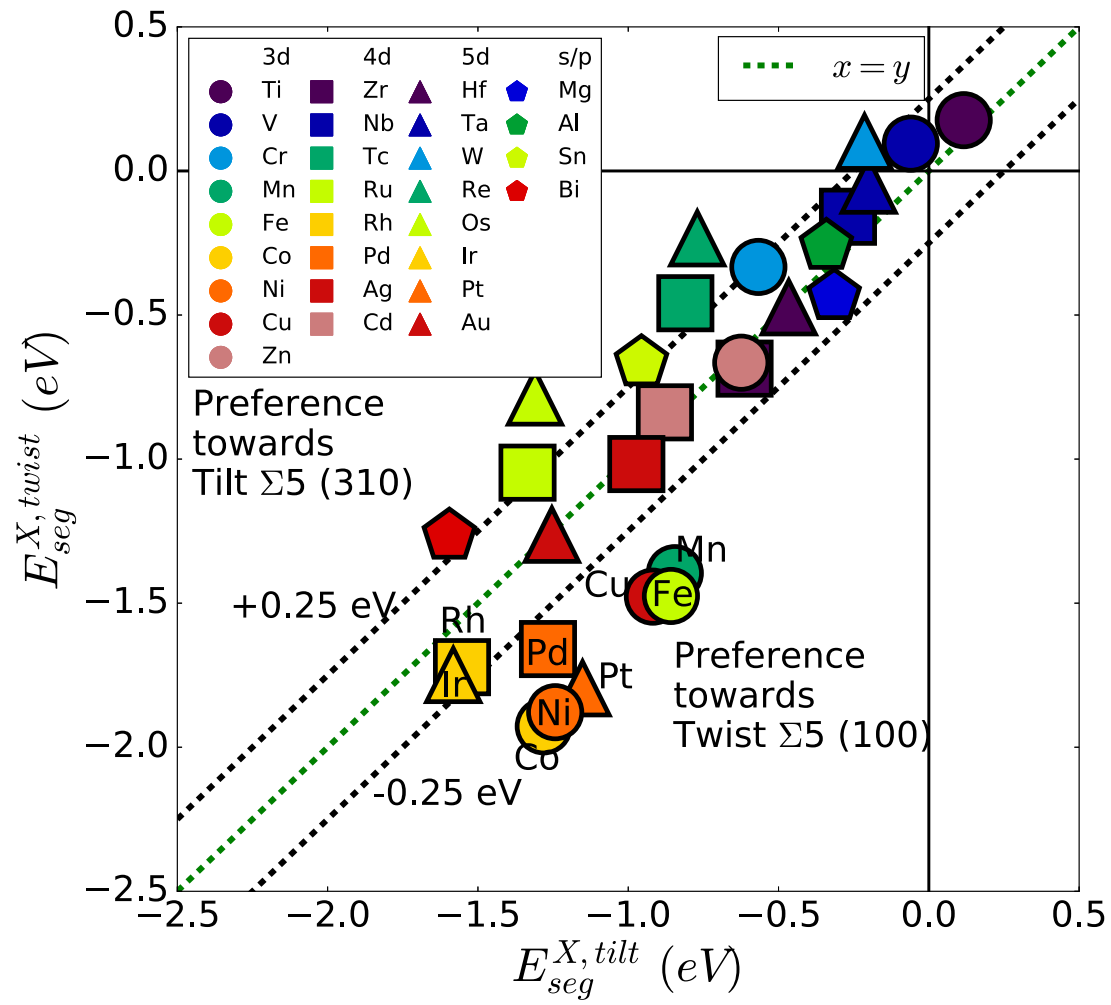

Figure 4: Comparison between the lowest $E_{\text {seg }}^{\mathrm{X}}$ of the tilt and twist GBs of Mo. 


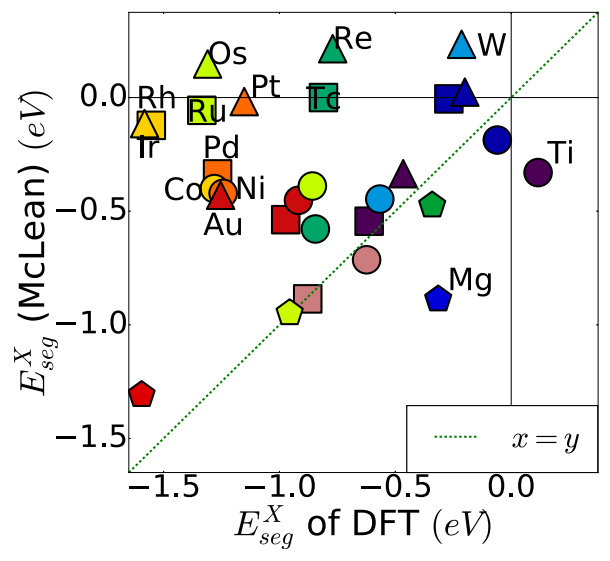

(a) McLean vs DFT

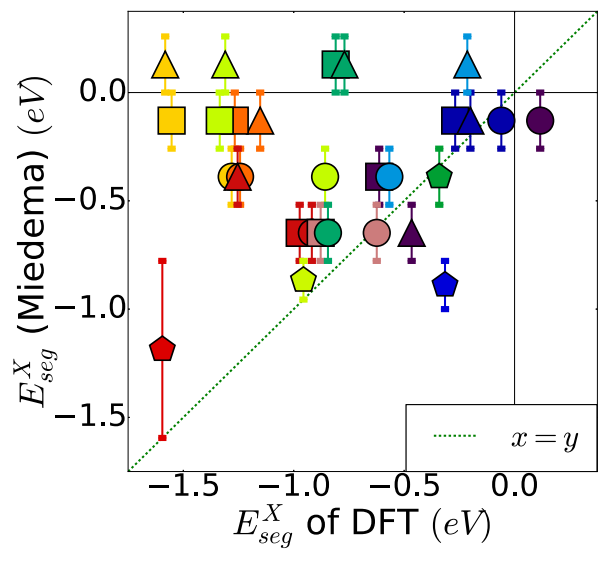

(b) Miedema vs DFT

$$
\begin{aligned}
& 3 d \bigcirc \mathrm{Ti} \bigcirc \mathrm{V} \bigcirc \mathrm{Cr} \bigcirc \mathrm{Mn} \bigcirc \mathrm{Fe} \bigcirc \mathrm{Co} \bigcirc \mathrm{Ni} \bigcirc \mathrm{Cu} \bigcirc \mathrm{Zn} \\
& \text { 4d } \square \mathrm{Zr} \square \mathrm{Nb} \square \mathrm{Tc} \square \mathrm{Ru} \square \mathrm{Rh} \square \mathrm{Pd} \square \mathrm{Ag} \square \mathrm{Cd} \\
& 5 \mathrm{~d} \Delta \mathrm{Hf} \Delta \mathrm{Ta} \Delta \mathrm{W} \Delta \mathrm{Re} \Delta \mathrm{Os} \Delta \mathrm{Ir} \Delta \mathrm{Pt} \triangle \mathrm{Au} \\
& \mathrm{s} / \mathrm{p} \triangle \mathrm{Mg} \triangle \mathrm{Al} \cap \mathrm{Sn} \triangle \mathrm{Bi}
\end{aligned}
$$

Figure 5: Comparison of the $E_{\text {seg }}^{\mathrm{X}}$ calculated using (a) the McLean model and (b) the Miedema model with the lowest DFT predicted values for the $\Sigma 5(310)$ tilt GB. For the Miedema model, $E_{\text {seg }}^{\mathrm{X}}$ is given as a range, and $E_{\text {seg }}^{\mathrm{X}}$ for $\mathrm{Bi}, \mathrm{Mg}$ and $\mathrm{Sn}$ have no minimum value stated in the literature [21]. 


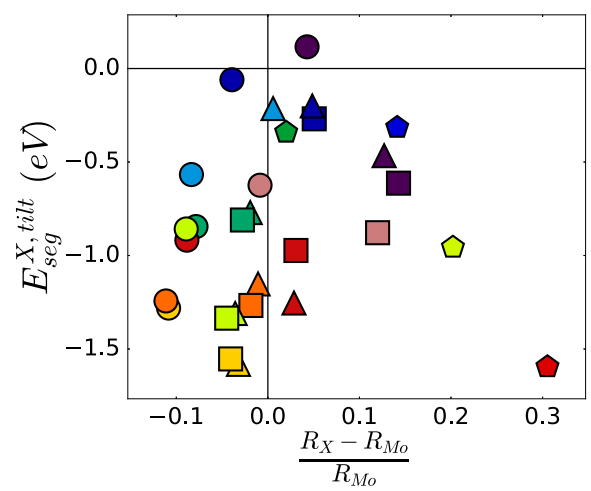

(a)

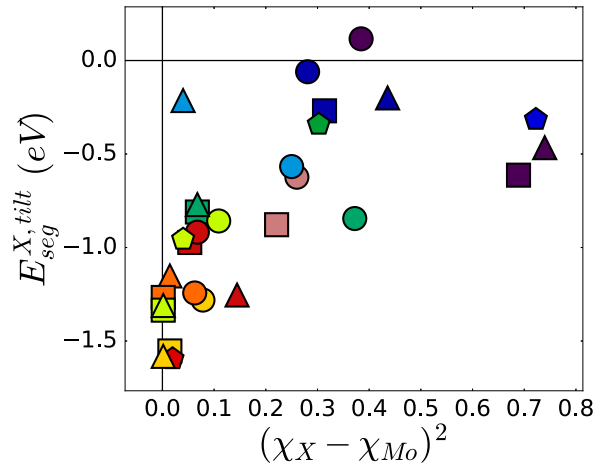

(b)

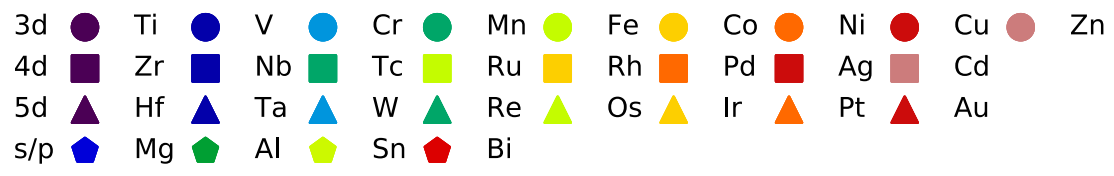

Figure 6: Plot of the lowest tilt segregation energy $E_{\text {seg }}^{\mathrm{X}}$ against (a) the relative metallic radius difference $\frac{R_{X}-R_{M o}}{R_{M o}}$ for each dopant $X$, and (b) the square of the relative Pauling electronegativity $\left(\chi_{\mathrm{X}}-\chi_{\mathrm{Mo}}\right)^{2}$ for each dopant $X$.

Re, Os and Ir would strengthen the Mo tilt GB. Using the m-to-s approach, only $\mathrm{Ta}$, Re, Os and $\mathrm{W}$ are predicted to have a weak strengthening effect, and all other dopants are predicted to embrittle or have no effect on the GB. Among the dopants that embrittle Mo, Bi has by far the greatest tendency to segregate and the most positive $E_{S E}^{\mathrm{X}}$.

195 Instead of the traditional one-factor bond-breaking model that relates $E_{S E}^{\mathrm{X}}$ with relative cohesive energy $\Delta E_{c o h}^{\mathrm{X}}[41,21]$, we investigated the following twofactor linear model:

$$
E_{S E}^{\mathrm{X}}=k_{c o h} \Delta E_{c o h}^{\mathrm{X}}+k_{R} \frac{R_{X}-R_{M o}}{R_{M o}}
$$

where $\frac{R_{X}-R_{M o}}{R_{M o}}$ is the relative metallic radii (a measure of strain), $\Delta E_{c o h}^{\mathrm{X}}$ is the relative difference in cohesive energy, and $k_{c o h}$ and $k_{R}$ are constant coefficients fitted using least squares regression.

We find that this two-factor model is able to account for most of the variation in the $E_{S E}^{\mathrm{X}}\left(R^{2}>0.79\right.$, see Figure 8), though the two coefficients differ significantly depending on whether the l-to-l or m-to-s approach is used in determining $E_{S E}^{\mathrm{X}}$. For the l-to-l approach (Figure 8(a)), the relative radii accounted for a ${ }_{205}$ much larger proportion of the $E_{S E}^{\mathrm{X}}\left(k_{R}=7.37 \pm 0.578\right)$, and the contribution of $\Delta E_{c o h}^{\mathrm{X}}, k_{c o h}$, is much smaller than the $1 / 3$ expected from the Seah bondbreaking model. For the m-to-s approach (Figure 8(b)), $k_{c o h}=0.39 \pm 0.042$, 
which is close to the $1 / 3$ value expected from the traditional bond-breaking model of Seah [41], though the relative radii still accounts for a significant proportion of the variation in $E_{S E}^{\mathrm{X}}$.

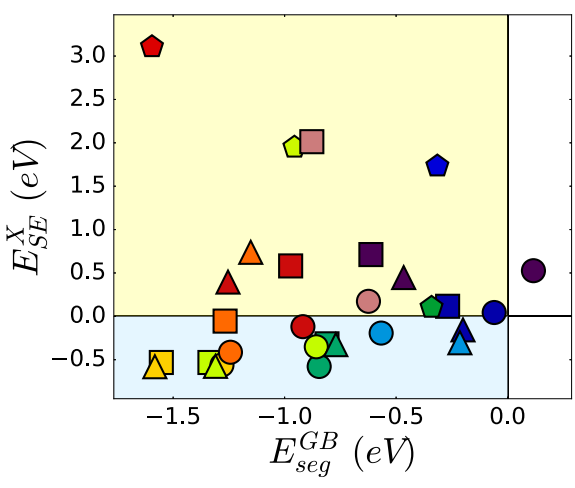

(a)

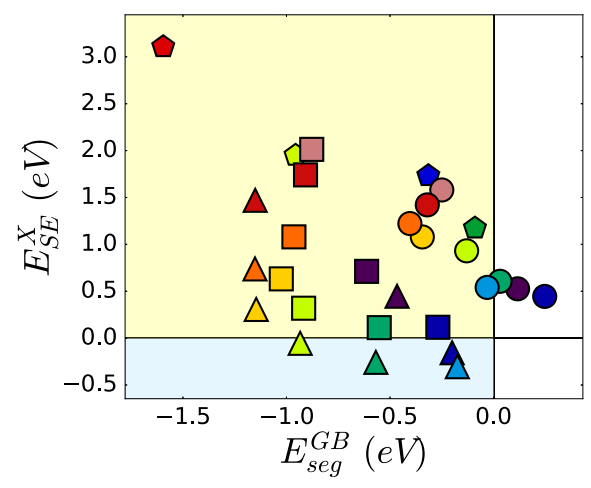

(b)

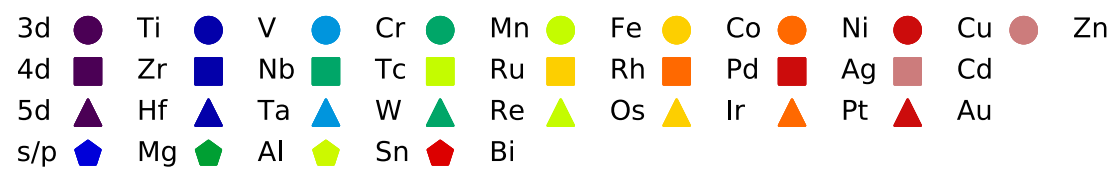

Figure 7: Plots of the strengthening energy $E_{S E}^{\mathrm{X}}$ versus segregation energy $E_{\text {seg }}^{\mathrm{X}}$ for the 29 dopants in the $\Sigma 5$ (310) tilt GB. (a) based on lowest energy dopant site in GB and free surface (l-to-l approach); (b) based on Site 0 (m-to-s approach). Dopants in the white region (positive $E_{\text {seg }}^{\mathrm{X}}$ ) prefer to stay in the bulk. For dopants that segregate, those with negative $E_{S E}^{\mathrm{X}}$ (blue region) tend to strengthen the GB.

\section{Discussion}

The segregation and strengthening/embrittling effects of dopants on Mo GB are of fundamental importance in a wide variety of applications. In this work, we performed a comprehensive DFT investigation of these effects for 29 dopants in the symmetric $\Sigma 5(310)$ tilt and $\Sigma 5(100)$ twist GBs of Mo.

We find that the type of GB generally has a small effect on the DFTpredicted dopant segregation energy. This is likely because both the twist and tilt GBs studied in this work are $\Sigma 5$ GBs with GB sites of relatively similar sizes (see Table 1). For the twist GB, most dopants prefer to segregate to the non-coincidental sites, which is in good agreement with the experimental work of Pénisson and Vystavel [42], which showed that Ni in Mo GBs prefer to segregate into random and high $\Sigma$ Mo GBs where there are more non-coincident sites for dopants to segregate into. We recognize that the behavior of general (random or high $\Sigma$ ) GBs can be somewhat different; yet the relative trends 225 of segregation for 29 dopants at two special $\Sigma 5$ (twist and tilt) GBs can still 


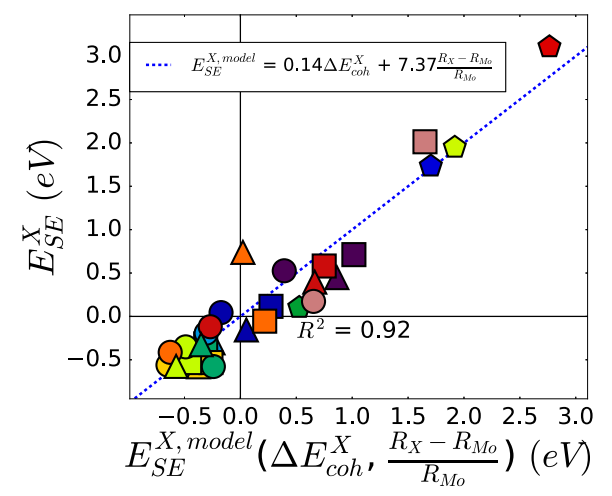

(a)

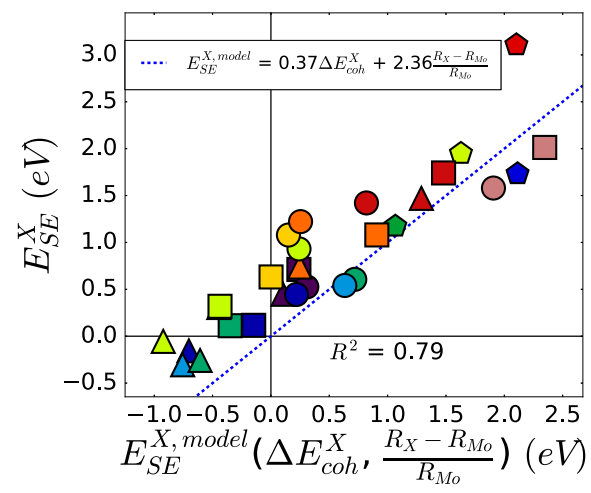

(b)

$$
\begin{aligned}
& 3 d \bigcirc \mathrm{Ti} \bigcirc \mathrm{V} \bigcirc \mathrm{Cr} \bigcirc \mathrm{Mn} \bigcirc \mathrm{Fe} \bigcirc \mathrm{Co} \bigcirc \mathrm{Ni} \bigcirc \mathrm{Cu} \bigcirc \mathrm{Zn} \\
& \text { 4d } \square \mathrm{Zr} \square \mathrm{Nb} \square \mathrm{Tc} \square \mathrm{Ru} \square \mathrm{Rh} \square \mathrm{Pd} \square \mathrm{Ag} \square \mathrm{Cd} \\
& 5 \mathrm{~d} \Delta \mathrm{Hf} \Delta \mathrm{Ta} \Delta \mathrm{W} \Delta \operatorname{Re} \Delta \text { Os } \Delta \text { Ir } \triangle \text { Pt } \Delta \mathrm{Au} \\
& \mathrm{s} / \mathrm{p} \triangle \mathrm{Mg} \triangle \mathrm{Al} \cap \mathrm{Sn} \triangle \mathrm{Bi}
\end{aligned}
$$

Figure 8: Plot of the observed strengthening energy $E_{S E}^{\mathrm{X}}$ for the site with the lowest $E_{\text {seg }}^{G B}$ versus the two factor linear model for $E_{S E}^{\mathrm{X}, \operatorname{model}}\left(E_{c o h}^{X}, \frac{R_{X}-R_{M o}}{R_{M o}}\right)$ for the 29 dopants in the $\Sigma 5$ (310) tilt GB. (a) Fitting performed based on the l-to-l approach. $k_{c o h}=0.14 \pm 0.023, k_{R}=$ $7.37 \pm 0.578$. (b) Fitting performed based on the m-to-s approach. $k_{c o h}=0.39 \pm 0.042, k_{R}=$ $2.40 \pm 1.076$. In all fittings, the $p$-value for all coefficients are $<0.05$. 
represent some useful trends in relative segregation behaviors of different alloy elements.

Historically, dopant segregation is attributed to the effect of strain and/or bond breaking arguments based on simple lattice models, which forms the basis for the McLean [28] and Miedema models [27]. We find significant disagreements between the more rigorous DFT segregation energies and those predicted by these empirical models for certain chemical classes, most notably Re, Os, the noble metals and the $3 d$ transition metals. The dopants for which there is the most significant disagreement are those that tend to form intermetallic 235 compounds with Mo. For example, Re, Os, Co, Pt, Ni, Ir, Pt, Tc, Zr, Hf, and Fe form at least one intermetallic compound with Mo [43], and the empirical models significantly underestimate the magnitude of the segregation energy for these dopants. Elements for which the empirical models are in good agreement with the DFT results, such as $\mathrm{W}, \mathrm{Nb}, \mathrm{Cr}, \mathrm{Cd}$, Ta, and $\mathrm{V}$, generally do not form intermetallic compounds with Mo. Precipitation of intermetallics at the two-dimensional GB is a precursor to bulk formation and is a chemical effect not accounted for in the empirical models. In Figure S3 of the Supplementary Information, we have plotted the $E_{\text {seg }}^{X, t i l t}$ against experimental solubilities of the dopant $\mathrm{X}$ in Mo. In general, the same qualitative trend is observed wherein 245 dopants with higher solubility have lower $E_{\text {seg }}^{X, t i l t}$, in agreement with the model proposed by Hondros et al. [44]. Moreover, it has been demonstrated generally for many metallic and ceramic materials (see several reviews by Cantwell et al. [22], Kaplan et al. [45], Harmer [46], Luo [47] and references therein) and specifically for several binary $[13,48]$ and ternary Mo based alloys $[49,50]$ as well as W-based alloys [51, 52] that GBs can undergo 2-D phase-like structural transitions, which are more likely to form in systems with strong, negative mixing enthalpies. These systems also tend to form intermetallic compounds and have less agreement with the current DFT computations, which do not consider interfacial structural transitions.

255 Similarly Seah [41], argued that the strengthening effect of dopants on GBs can be modeled with a simple bond-breaking argument, and is related to the difference in cohesive energies. More recently Gibson and Schuh [21], performed a survey of first principles calculations to show that segregation-induced grain boundary embrittlement is generally predicted by bond-breaking arguments. While the general trends observed in this work support these previous findings, we find that dopant site preference also has a significant impact on the relative influence of relative radii $\frac{R_{X}-R_{M o}}{R_{M o}}$ and relative cohesive energies $\Delta E_{c o h}^{\mathrm{X}}$ on the $E_{S E}^{\mathrm{X}}$. In general, the relative radius drives site preference, more so in the GB than in the free surface. As a result, a model that assumes cleavage of the lowest energy tilt GB configuration to form the lowest energy free surface configuration (Figure 8(a)) finds a weak dependence of $E_{S E}^{\mathrm{X}}$ on $\Delta E_{c o h}^{\mathrm{X}}$, and a stronger dependence on $\frac{R_{X}-R_{M o}}{R_{M o}}$. On the other hand, a model that assumes cleavage of a tilt GB with the dopant at the mirror plane site to form a free surface with the dopant on the corresponding surface site shows a much stronger depen${ }_{270}$ dence of $E_{S E}^{\mathrm{X}}$ on $\Delta E_{c o h}^{\mathrm{X}}$, which is in line with the bond-breaking model [41]. In 
essence, the bond-breaking argument applies when the dopant site environment remains consistent between the GB and free surface, i.e., at the mirror plane in the tilt GB or either of the investigated sites in the twist GB. We also recognize that the bond-breaking arguments may work (even) better with general random/non-lattice-matching or high $\Sigma$ ) GBs where bonds are less satisfied.

There are some disagreements between the predicted $E_{S E}^{\mathrm{X}}$ using the l-to1 approach and previous experimental data. For instance, the predicted $E_{S E}^{\mathrm{X}}$ suggests that $\mathrm{Ni}$ is a good candidate dopant for strengthening Mo GBs, but experimentally, it is well-known that Ni tends to embrittle Mo GBs. More recent experimental and phenomenological thermodynamic modeling studies find that the mechanism leading to the embrittlement of Mo GBs due to Ni doping is likely due to the formation of premelting-like (disorderd) complexions (i.e. 2D interfacial phases) [13, 48]. While Hwang and Huang [53, 14, 54] earlier proposed the formation of $\delta$-NiMo intermetallic compounds at the Mo GB as the cause of embrittlement, more recent work by one of the co-authors of the present work has shown that $\delta$-NiMo intermetallic compounds does not wet the general GBs of Mo, as the dihedral angles are $>100^{\circ}$; yet, a nanoscale disordered, Ni-enriched interfacial complexion was observed in well-quenched specimens [13]. Thus, it is possible that those $\delta$-NiMo intermetallic compounds 290 formed via recrystallization of disordered complexions which were formed at high temperatures. This can also be a contributing factor to the embrittlement of slowly-cooled Mo-Ni specimens, making Hwang and Huang's observations relevant to the embrittlement mechanism. These studies can adequately explain the discrepancy between the DFT predictions and the experimental data, since the current DFT calculations do not consider interfacial disordering or structural transitions.

We also note that the alternative m-to-s model predicts that Ni embrittles the Mo GB which is consistent with experimental observations. For a rapid fracture process where atoms do not have the time to equilibrate to the preferred 300 low energy sites, the m-to-s model may be more appropriate. Moreover, the DFT predictions (whether l-to-l or m-to-s) are premised on a simple cleavage model where a GB separates to form two free surfaces. Such a model would not be appropriate for a mechanism of embrittlement via intermetallic compound formation. Finally, previous experimental work suggests that $\mathrm{Ni}$ tends to segregate at random and high $\Sigma$ Mo GBs. The GBs investigated in this work are low $\Sigma$, which may present different dopant site energetics. It is also known that general or high $\Sigma$ GBs are more prone to disorder at high temperatures [47].

Based on the m-to-s model for the tilt GB, only Re, Os, Ta and W are predicted to have a weak strengthening effect on Mo. For the $\Sigma 5(100)$ twist $\mathrm{GB}, \mathrm{Mn}, \mathrm{Fe}$, $\mathrm{Co}$ and $\mathrm{Nb}$ are predicted to have reasonable strengthening effects (see Figure S4 in the SI). Re is also shown to have a weak strengthening effect in the twist GB. This is in agreement with previous experiments that have shown the alloying of Re with Mo and other metals will improve their mechanical properties [55, 56, 57]. 


\section{Conclusion}

In conclusion, we have studied the segregation and strengthening effects of 29 metallic dopants on the $\Sigma 5(100)$ twist and $\Sigma 5(310)$ tilt GB in Mo using DFT calculations. In comparison with the empirical McLean and Miedema models, DFT predicts significantly larger in magnitude segregation energies for the noble 320 and $3 d$ transition metals, especially those that are known to form intermetallic compounds with Mo. In terms of the strengthening energy, we find that dopant site preference and strain effects have a significant influence, in addition to the difference in cohesive energy based on traditional bond-breaking considerations. Assuming a fast cleavage model for fracture, only Ta Re, Os and W are predicted 325 to have a weak strengthening effect on Mo for the $\Sigma 5(310)$ tilt GB, and Mn, $\mathrm{Fe}, \mathrm{Co}$ and $\mathrm{Nb}$ are predicted to have reasonable strengthening effects for the $\Sigma 5(100)$ twist GB.

\section{Acknowledgement}

This work was supported by the U.S. Department of Defense as a part of the

330 National Security Science and Engineering Faculty Fellowship (NSSEFF) under ONR Grant No.N00014-15-1-0030. The authors also gratefully acknowledge computing resources provided by the Extreme Science and Engineering Discovery Environment (XSEDE), which is supported by National Science Foundation grant number ACI-1053575.

\section{References}

[1] J. Che, C. Chan, W.-E. Jian, T. Leung, Surface atomic structures, surface energies, and equilibrium crystal shape of molybdenum, Physical Review B 57 (1998) 1875-1880.

[2] J. Shields, Applications of Mo Metal and Its Alloys, 2013.

345 [3] R. Causey, C. Kunz, D. Cowgill, Deuterium retention and release from molybdenum exposed to a Penning discharge, Journal of Nuclear Materials 337-339 (2005) 600-603.

[4] J. Schlichting, Molybdenum disilicide as a component of modern hightemperature composites, INIS 10 (1978). 
[5] N. S. Rasor, J. D. Mcclelland, A. International, C. Park, Thermal Properties of Graphite, Molybdenum, and Tantalum to their Destruction Temperatures, J. Phys. Chem. Solids 15 (1960) 17-26.

[6] H. C. Hsu, S. C. Wu, S. K. Hsu, T. Y. Chang, W. F. Ho, Effect of ball milling on properties of porous Ti-7.5Mo alloy for biomedical applications, Journal of Alloys and Compounds 582 (2014) 793-801.

[7] M. Xia, T. Lei, N. Lv, N. Li, Synthesis and electrocatalytic hydrogen evolution performance of $\mathrm{NiMoCu}$ alloy coating electrode, International Journal of Hydrogen Energy 39 (2014) 4794-4802.

[8] G. Liu, G. J. Zhang, F. Jiang, X. D. Ding, Y. J. Sun, J. Sun, E. Ma, Nanostructured high-strength molybdenum alloys with unprecedented tensile ductility., Nature materials 12 (2013) 344-50.

[9] T. Watanabe, S. Tsurekawa, Toughening of brittle materials by grain boundary engineering, Materials Science and Engineering A 387-389 (2004) 447-455.

[10] J. R. Rice, J. S. Wang, Embrittlement of interfaces by solute segregation, Materials Science and Engineering A 107 (1989) 23-40.

[11] G. Duscher, M. F. Chisholm, U. Alber, M. Rühle, Bismuth-induced embrittlement of copper grain boundaries., Nature materials 3 (2004) 621-6.

[12] R. Schweinfest, A. T. Paxton, M. W. Finnis, Bismuth embrittlement of copper is an atomic size effect., Nature 432 (2004) 1008-11.

[13] X. Shi, J. Luo, Grain boundary wetting and prewetting in Ni-doped Mo, Applied Physics Letters 94 (2009) 11-13.

[14] K. Hwang, H. Huang, Identification of the Segregation Layer and its Effects on the Activated Sintering and Ductility of Ni-Doped Molybdenum, Acta materialia 51 (2003) 3915-3926.

[15] M. K. Miller, E. a. Kenik, M. S. Mousa, K. F. Russell, a. J. Bryhan, Improvement in the ductility of molybdenum alloys due to grain boundary segregation, Scripta Materialia 46 (2002) 299-303.

[16] A. Charai, I. Kutcherinenko, J. Pbnisson, V. Pontikis, L. Priester, K. Wolski, T. Vystavel, Electron microscopy and Auger spectroscopy study of the wetting of the grain boundaries in the systems Mo-Pb , Mo-Sn, Mo-Ni and Ni-Pb, Journal de Physique IV 12 (2002) 277-87.

[17] R. Janisch, C. Elsässer, Segregated light elements at grain boundaries in niobium and molybdenum, Physical Review B 67 (2003) 224101.

[18] L. Sun, S. Jin, H.-B. Zhou, Y. Zhang, G.-H. Lu, Dissolution and diffusion of hydrogen in a molybdenum grain boundary: A first-principles investigation, Computational Materials Science 102 (2015) 243-249. 
[19] A. Cotrell, Strengthening of grain boundaries by segregated interstitials in iron, Materials Science and Technology 6 (1990) 121-123.

390

[20] L. Pavel, Grain boundary segregation in metals, 2008. URL: http://medcontent .metapress . com/index/A65RM03P4874243N . pdf. doi:10.1007/978-3-642-12505-8.

[21] M. a. Gibson, C. a. Schuh, A survey of ab-initio calculations shows that segregation-induced grain boundary embrittlement is predicted by bondbreaking arguments, Scripta Materialia 113 (2016) 55-58.

[22] P. R. Cantwell, M. Tang, S. J. Dillon, J. Luo, G. S. Rohrer, M. P. Harmer, Grain boundary complexions, Acta Materialia 62 (2014) 1-48.

[23] H. Jin, I. Elfimov, M. Militzer, Study of the interaction of solutes with ??5 (013) tilt grain boundaries in iron using density-functional theory, Journal of Applied Physics 115 (2014).

[24] Z.-W. Li, X.-S. Kong, C.-S. Liu, Q.-F. Fang, Segregation of alloying atoms at a tilt symmetric grain boundary in tungsten and their strengthening and embrittling effects, Chinese Physics B 23 (2014) 106107.

[25] V. I. Razumovskiy, A. Y. Lozovoi, I. M. Razumovskii, ScienceDirect Firstprinciples-aided design of a new Ni-base superalloy : Influence of transition metal alloying elements on grain boundary and bulk cohesion, Acta Materialia 82 (2015) 369-377.

[26] W. Liu, H. Han, C. Ren, H. Yin, Y. Zou, P. Huai, H. Xu, Effects of rareearth on the cohesion of $\mathrm{Ni} \Sigma 5$ (012) grain boundary from first-principles calculations, Computational Materials Science 96 (2015) 374-378.

[27] A. Miedema, Surface segregation in alloys of transition-metals, Zeitschrift Fur Metallkunde 7 (1978) 455-461.

[28] D. McLean, Grain Boundaries in Metals, Oxford University Press, 1957.

[29] S. P. Ong, W. D. Richards, A. Jain, G. Hautier, M. Kocher, S. Cholia, D. Gunter, V. L. Chevrier, K. A. Persson, G. Ceder, Python Materials Genomics (pymatgen): A robust, open-source python library for materials analysis, Computational Materials Science 68 (2013) 314-319.

[30] a. Brokman, R. W. Balluffi, Coincidence lattice model for the structure and energy of grain boundaries, Acta Metallurgica 29 (1981) 1703-1719.

[31] G. Kresse, J. Furthmüller, Efficient iterative schemes for ab initio totalenergy calculations using a plane-wave basis set., Physical review. B, Condensed matter 54 (1996) 11169-11186.

[32] W. Kohn, L. J. Sham, Self-Consistent Equations INcluding Exchange and Correlation Effects*, Physical Review B 140 (1965). 
[33] J. Kresse, G., Furthmuller, Software VASP, Vienna, Physical Review B 54 (1996) 169.

[34] P. E. Blöchl, Projector augmented-wave method, 1994. doi:10.1103/ PhysRevB.50.17953.

[35] J. Perdew, K. Burke, M. Ernzerhof, Generalized Gradient Approximation Made Simple., Physical review letters 77 (1996) 3865-3868.

[36] M. Methfessel, A. T. Paxton, High-precision sampling for Brillouin-zone integration in metals, Physical Review B 40 (1989) 3616-3621.

[37] S. Johnson, J. Joannopoulos, Block-iterative frequency-domain methods for Maxwell's equations in a planewave basis., Optics Express 8 (2001) 173-190.

[38] R. Kirchheim, B. Somerday, P. Sofronis, Chemomechanical effects on the separation of interfaces occurring during fracture with emphasis on the hydrogen-iron and hydrogen-nickel system, Acta Materialia 99 (2015) 8798.

[39] J. Friedel, Electronic structure of primary solid solutions in metals, Adv. Phys. 50 (2001) 539-595.

[40] L. Pauling, The Nature of the Chemical Bond. IV. The Energy of Single Bonds and the Relative Electronegativity of Atoms, Journal of the American Chemical Society 481 (1932) 3570-3582.

[41] M. Seah, Adsorption-induced interface decohesion, Acta Metallurgica 28 (1980) 955-962.

[42] J. Pénisson, T. Vystavel, Wetting of molybdenum grain boundaries by nickel: effect of the boundary structure and energy, Acta Materialia 48 (2000) 3303-3310.

[43] A. International, A-B-C Phase Diagram, ASM Alloy Phase Diagrams Database, 2006. URL: http://www1.asminternational.org/ AsmEnterprise/APD.

[44] E. Hondros, M. Seah, S. Hofmann, C. Lejček, No Title, in: R. Cahn, P. Haasen (Eds.), Physical Metallurgy, North Holland, Amsterdam, 1996, pp. 1201-1289.

[45] W. D. Kaplan, D. Chatain, P. Wynblatt, W. C. Carter, A review of wetting versus adsorption, complexions, and related phenomena: The rosetta stone of wetting, Journal of Materials Science 48 (2013) 5681-5717.

[46] M. P. Harmer, The Phase Behavior of Interfaces, Science 332 (2011) 182183. 
[47] J. Luo, Developing Interfacial Phase Diagrams for Applications in Activated Sintering and Beyond: Current Status and Future Directions, Journal of the American Ceramic Society 95 (2012) 2358-2371.

[48] X. Shi, J. Luo, Developing grain boundary diagrams as a materials science tool: A case study of nickel-doped molybdenum, Physical Review B Condensed Matter and Materials Physics 84 (2011) 1-14.

[49] N. Zhou, J. Luo, Developing grain boundary diagrams for multicomponent alloys, Acta Materialia 91 (2015) 202-216.

[50] J. I. Jung, N. Zhou, J. Luo, Effects of sintering aids on the densification of Mo-Si-B alloys, Journal of Materials Science 47 (2012) 8308-8319.

[51] J. Luo, V. K. Gupta, D. H. Yoon, H. M. Meyer, Segregation-induced grain boundary premelting in nickel-doped tungsten, Applied Physics Letters 87 (2005) 1-3.

[52] V. K. Gupta, D. H. Yoon, H. M. Meyer, J. Luo, Thin intergranular films and solid-state activated sintering in nickel-doped tungsten, Acta Materialia 55 (2007) 3131-3142.

[53] K. S. Hwang, H. S. Huang, Liquid phase sintering of molybdenum with Ni and $\mathrm{Cu}$ additions, Materials Chemistry and Physics 67 (2001) 92-100.

[54] K. S. Hwang, H. S. Huang, Ductility improvement of Ni-added molybdenum compacts through the addition of $\mathrm{Cu}$ and Fe powders, International Journal of Refractory Metals and Hard Materials 22 (2004) 185-191.

[55] J.-H. Liao, H.-Y. Bor, C.-G. Chao, T.-F. Liu, Effects of Rhenium on Microstructure and Phase Stability of MAR-M247 Ni-Base Fine-Grain Superalloy, Materials Transactions 51 (2010) 810-817.

[56] J.-H. Liao, H.-Y. Bor, C.-G. Chao, T.-F. Liu, Influence of Rhenium on the Mechanical Behavior and Fracture Mechanism of a Fine-Grain Superalloy at Elevated Temperatures, Materials Transactions 52 (2011) 201-209.

[57] N. Igata, A. Kohyama, K. Itadani, The effect of rhenium on some properties of molybdenum alloys as fusion reactor material, Journal of Nuclear Materials 85-86 (1979) 895-899. 